$\mathrm{DE}$

M E D I C I N A

T R O P I C A L

$\mathrm{DE}$

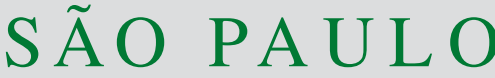

JOURNAL OF THE SÃO PAULO INSTITUTE OF TROPICAL MEDICINE

${ }^{1}$ University of Kragujevac, Faculty of Medical Sciences, Department of Pharmacology and toxicology, Kragujevac, Serbia

2University of Kragujevac, Faculty of Medical Sciences, Department of Anatomy, Kragujevac, Serbia

${ }^{3}$ University of Kragujevac, Faculty of Medical Sciences, Department of Pharmacy, Kragujevac, Serbia

${ }^{4}$ Private Policlinic Center Eurofar Sarajevo, Cardiology Department, Sarajevo, Bosnia and Herzegovina

${ }^{5}$ University of Priština, School of Medicine, Department of Infectious Diseases, Pristina, Serbia

Correspondence to: Jovana Z.

Milosavljevic

University of Kragujevac, Faculty of Medical Sciences, Department of Anatomy, Svetozara Markovica 69, 34000 Kragujevac, Serbia

Tel: +38 1669178800

E-mail: jowana.ilic@yahoo.com

Received: 17 March 2021

Accepted: 7 May 2021

\section{Antimicrobial treatment of Corynebacterium striatum invasive infections: a systematic review}

\author{
Milos N. Milosavljevic ${ }^{\circledR 1}$, Jovana Z. Milosavljevic ${ }^{(1)}$, Aleksandar G. Kocovic $^{3}$, \\ Srdjan M. Stefanovic ${ }^{3}$, Slobodan M. Jankovic ${ }^{1}$, Miralem Djesevic ${ }^{4}$, Milica N. \\ Milentijevic $^{5}$
}

\section{ABSTRACT}

The aim of this study was to establish an evidence-based guideline for the antibiotic treatment of Corynebacterium striatum infections. Several electronic databases were systematically searched for clinical trials, observational studies or individual cases on patients of any age and gender with systemic inflammatory response syndrome, harboring $C$. striatum isolated from body fluids or tissues in which it is not normally present. $C$. striatum had to be identified as the only causative agent of the invasive infection, and its isolation from blood, body fluids or tissues had to be confirmed by one of the more advanced diagnostic methods (biochemical methods, mass spectrometry and/or gene sequencing). This systematic review included 42 studies that analyzed 85 individual cases with various invasive infections caused by C. striatum. More than one isolate of $C$. striatum exhibited $100 \%$ susceptibility to vancomycin, linezolid, teicoplanin, piperacillin-tazobactam, amoxicillin-clavulanate and cefuroxime. On the other hand, some strains of this bacterium showed a high degree of resistance to fluoroquinolones, to the majority majority of $\beta$-lactams, aminoglycosides, macrolides, lincosamides and cotrimoxazole. Despite the antibiotic treatment, fatal outcomes were reported in almost $20 \%$ of the patients included in this study. Gene sequencing methods should be the gold standard for the identification of $C$. striatum, while MALDI-TOF and the Vitek system can be used as alternative methods. Vancomycin should be used as the antibiotic of choice for the treatment of $C$. striatum infections, in monotherapy or in combination with piperacillintazobactam. Alternatively, linezolid, teicoplanin or daptomycin may be used in severe infections, while amoxicillin-clavulanate may be used to treat mild infections caused by $C$. striatum.

KEYWORDS: Corynebacterium striatum. Invasive infection. Antibiotic treatment. Systematic review.

\section{INTRODUCTION}

Corynebacterium striatum is a non-diphtheric gram-positive, aerobic and facultative anaerobic, non-sporulating and immobile bacillus ${ }^{1,2}$. Although this bacterium is a normal resident of skin and mucous membranes in humans, a large number of different invasive infections caused by this species have been reported in the last two decades ${ }^{3}$. C. striatum causes serious infections primarily in immunocompromised patients, such as those in the terminal stage of cancer and presenting with other critical conditions ${ }^{4}$. In addition, invasive diagnostic and therapeutic procedures, long-term use of broad-spectrum antibiotics and prolonged hospitalization were also identified as relevant risk factors for $C$. striatum infections ${ }^{5,6}$. However, there is a growing evidence that $C$. striatum can cause serious 
infections in immunocompetent hosts, as well ${ }^{6}$. In addition, cases of patient-to-patient spreading of $C$. striatum with serious nosocomial outbreaks are described in intensive care units. Therefore, in many countries, nowadays, $C$. striatum is considered as an emerging pathogen ${ }^{7,8}$.

To the best of our knowledge, no evidence-based guidelines have been proposed to date to support an adequate antibiotic therapy for $C$. striatum infections. Only one review summarized the combination of amoxicillin and rifampin in the treatment of bone and joint infections ${ }^{9}$. Current evidence suggests certain antibiotics for the treatment of these infections based on in vitro susceptibility testing ${ }^{10,11}$. The resistance of $C$. striatum to a large number of antimicrobial agents used to treat gram-positive infections, such as penicillin, ceftriaxone, meropenem, clindamycin and tetracycline, has been deeply studied ${ }^{10}$. Since the causal role of C. striatum in the development of different types of invasive infections is undeniable, the doses and duration of antibiotic therapy can vary significantly. Given all of the above, there is a growing need to establish evidence-based guidelines for the antibiotic treatment of C. striatum infections.

Therefore, based on the available literature evidence on previously successful antibiotic treatment for $C$. striatum infections, our attempt was to elaborate a guide that could help physicians to address this rare, although potentially serious problem in clinical practice.

\section{MATERIALS AND METHODS}

The research protocol was initially registered at PROSPERO database of systematic review articles and meta-analyzes (registration $\mathrm{N}^{\circ}$ CRD42020185512).

Different types of articles (case reports, case series, clinical trials, observational studies) were considered as data sources for this research. Patients of any gender or age were included in the systematic examination if they were infected with $C$. striatum. The bacterium had to be identified as the only causative agent of the invasive infection, and its isolation from blood, body fluids or tissues had to be confirmed by one of the more advanced diagnostic methods (Vitek 2, API or BD Phoenix biochemical systems, MALDI-TOF mass spectrometry and/or by 16s RNA sequencing).

Articles were excluded if the data of patients were incomplete, if $C$. striatum was not the only isolated microorganism (mixed infections), in case the full text of the article was not found or if the article was found to be irrelevant (C. striatum was only mentioned in the text). There were no additional restrictions on the language or date of publication.
The following electronic databases were searched: MEDLINE (PubMed), EBSCO (Discovery Service), SCOPUS, SCIndex (Serbian Citation Index), Cochrane database of published clinical trials - Central (Wiley Online Library), and ClinicalTrials.gov (U.S. National Library of Medicine). The database search included all studies with human participants published until November 15, 2020.

All authors, except for the senior investigator (SJ), independently searched for the mentioned databases, according to their own search strategy. MM's search strategy provided the highest number of results in the MEDLINE database:

("corynebacterium striatum"[Supplementary Concept] OR "corynebacterium striatum"[All Fields] OR “corynebacterium striatum”[All Fields]) AND (((“invasibility”[All Fields] OR "invasible”[All Fields] OR “invasion”[All Fields] OR "invasions”[All Fields] OR "invasive"[All Fields] OR "invasively"[All Fields] OR "invasiveness"[All Fields] OR "invasives"[All Fields] OR "invasivity"[All Fields]) AND ("infect"[All Fields] OR "infectability"[All Fields] OR "infectable"[All Fields] OR "infectant”[All Fields] OR "infectants"[All Fields] OR "infected"[All Fields] OR "infecteds"[All Fields] OR "infectibility”[All Fields] OR "infectible"[All Fields] OR "infecting”[All Fields] OR "infection s"[All Fields] OR "infections"[MeSH Terms] OR "infections"[All Fields] OR "infection"[All Fields] OR "infective"[All Fields] OR "infectiveness"[All Fields] OR "infectives"[All Fields] OR "infectivities"[All Fields] OR "infects"[All Fields] OR "pathogenicity"[MeSH Subheading] OR "pathogenicity"[All Fields] OR "infectivity"[All Fields])) OR ("endocarditis"[MeSH Terms] OR "endocarditis"[All Fields] OR "endocarditides"[All Fields]) OR ("osteomyelities"[All Fields] OR "osteomyelitis"[MeSH Terms] OR “osteomyelitis"[All Fields] OR "osteomyelitides"[All Fields]) OR ("arthritis"[MeSH Terms] OR "arthritis"[All Fields] OR “arthritides”[All Fields] OR "polyarthritides"[All Fields]) OR (("prosthetic"[All Fields] OR "prosthetically"[All Fields] OR "prosthetics"[All Fields]) AND ("joint s"[All Fields] OR "joints"[MeSH Terms] OR “joints”[All Fields] OR "joint”[All Fields]) AND ("infect"[All Fields] OR "infectability"[All Fields] OR "infectable"[All Fields] OR "infectant"[All Fields] OR "infectants"[All Fields] OR "infected"[All Fields] OR "infecteds"[All Fields] OR "infectibility”[All Fields] OR "infectible"[All Fields] OR "infecting"[All Fields] OR "infection s"[All Fields] OR "infections"[MeSH Terms] OR "infections"[All Fields] OR "infection"[All Fields] OR "infective"[All Fields] OR "infectiveness"[All Fields] OR "infectives"[All Fields] OR “infectivities”[All Fields] OR "infects"[All 
Fields] OR "pathogenicity"[MeSH Subheading] OR "pathogenicity"[All Fields] OR "infectivity"[All Fields])) OR (("joint s"[All Fields] OR "joints"[MeSH Terms] OR “joints”[All Fields] OR “joint”[All Fields]) AND ("infect"[All Fields] OR "infectability"[All Fields] OR "infectable"[All Fields] OR "infectant"[All Fields] OR “infectants"[All Fields] OR "infected"[All Fields] OR "infecteds"[All Fields] OR "infectibility"[All Fields] OR "infectible"[All Fields] OR "infecting"[All Fields] OR "infection s"[All Fields] OR "infections"[MeSH Terms] OR “infections"[All Fields] OR "infection"[All Fields] OR "infective"[All Fields] OR "infectiveness"[All Fields] OR “infectives"[All Fields] OR "infectivities"[All Fields] OR “infects”[All Fields] OR "pathogenicity"[MeSH Subheading] OR "pathogenicity"[All Fields] OR "infectivity”[All Fields])) OR ("synovitis"[MeSH Terms] OR “synovitis"[All Fields] OR "synovitides”[All Fields]) OR ("peritoneally"[All Fields] OR "peritoneum"[MeSH Terms] OR "peritoneum" [All Fields] OR "peritoneal”[All Fields] OR "peritonism"[All Fields] OR "peritonitis" [MeSH Terms] OR "peritonitis"[All Fields]) OR ((“peritoneal dialysis, continuous ambulatory"[MeSH Terms] OR ("peritoneal"[All Fields] AND "dialysis"[All Fields] AND "continuous"[All Fields] AND "ambulatory”[All Fields]) OR "continuous ambulatory peritoneal dialysis"[All Fields] OR “capd”[All Fields]) AND ("peritoneally”[All Fields] OR “peritoneum”[MeSH Terms] OR “peritoneum”[All Fields] OR "peritoneal”[All Fields] OR "peritonism"[All Fields] OR "peritonitis"[MeSH Terms] OR "peritonitis"[All Fields])) OR ("pneumonia"[MeSH Terms] OR "pneumonia"[All Fields] OR "pneumoniae"[All Fields] OR "pneumonias"[All Fields] OR "pneumoniae s"[All Fields]) OR ("sepsis”[MeSH Terms] OR "sepsis"[All Fields]) OR ("sepsis”[MeSH Terms] OR "sepsis”[All Fields] OR "septicaemias"[All Fields] OR "septicemias”[All Fields] OR “septicaemia”[All Fields] OR "septicemia”[All Fields]) OR ("bacteraemia"[All Fields] OR "bacteremia"[MeSH Terms] OR "bacteremia”[All Fields] OR "bacteraemias"[All Fields] OR "bacteremias"[All Fields]) OR ("meningeal"[All Fields] OR "meninges"[MeSH Terms] OR "meninges"[All Fields] OR "meninge"[All Fields] OR "meningism"[MeSH Terms] OR "meningism"[All Fields] OR "meningisms"[All Fields] OR "meningitis" [MeSH Terms] OR "meningitis"[All Fields] OR "meningitides"[All Fields]) OR ("abscess"[MeSH Terms] OR "abscess"[All Fields] OR “abscesses”[All Fields] OR “abscessation”[All Fields] OR "abscessed"[All Fields] OR "abscessing”[All Fields]) OR ((“central venous catheters"[MeSH Terms] OR ("central”[All Fields] AND "venous"[All Fields] AND "catheters"[All Fields]) OR "central venous catheters"[All Fields] OR ("central"[All Fields] AND "venous"[All
Fields] AND "catheter"[All Fields]) OR "central venous catheter"[All Fields]) AND ("family"[MeSH Terms] OR "family"[All Fields] OR "relation"[All Fields] OR "relatability"[All Fields] OR "relatable"[All Fields] OR "related"[All Fields] OR "relates"[All Fields] OR "relating"[All Fields] OR "relational"[All Fields] OR "relations"[All Fields]) AND ("infect"[All Fields] OR "infectability"[All Fields] OR "infectable"[All Fields] OR “infectant”[All Fields] OR "infectants”[All Fields] OR "infected"[All Fields] OR "infecteds"[All Fields] OR "infectibility”[All Fields] OR "infectible”[All Fields] OR "infecting"[All Fields] OR "infection s"[All Fields] OR “infections"[MeSH Terms] OR "infections”[All Fields] OR "infection"[All Fields] OR "infective"[All Fields] OR “infectiveness"[All Fields] OR "infectives"[All Fields] OR "infectivities"[All Fields] OR "infects"[All Fields] OR "pathogenicity”[MeSH Subheading] OR "pathogenicity”[All Fields] OR “infectivity”[All Fields])) OR ("catheter related infections"[MeSH Terms] OR ("catheter related"[All Fields] AND "infections"[All Fields]) OR "catheter related infections"[All Fields] OR ("catheter"[All Fields] AND "related"[All Fields] AND "infection"[All Fields]) OR “catheter related infection"[All Fields])).

All the selected articles were firstly evaluated on the basis of titles and abstracts. If it was not possible to make a decision in this way, the full-text article was analyzed. If the authors did not agree on the inclusion of an individual article in the systematic review, the senior researcher made the final decision.

From each article that met the inclusion criteria, the following data were extracted and inserted into an Excel file: (1) Publication ID, (2) Report ID, (3) Review author initials, (4) Citation and contact details, (5) Eligibility for review, (6) Study design, (7) Total study duration, (8) Risk of bias, (9) Total number of patients, (10) Age of patients, (11) Gender of patients, (12) Country, (13) Presence of hypertension, (14) Presence of diabetes, (15) Presence of a malignancy, (16) Postoperative infections, (17) Infections on embedded artificial materials, (18) Site of C. striatum isolation (body fluid or tissue), (19) Sampling method, (20) Method of $C$. striatum identification (biochemical methods and/or MALDI TOF and/or 16S RNA sequencing), (21) Maximal level of serum C-reactive protein (CRP) during the infection, (22) Maximal level of serum procalcitonin (PCT) during the infection, (23) Maximal white cells count (WBC) during the infection, (24) Diagnosis, (25) Morphological diagnoses that confirmed the invasive infection (NMR, ultrasound, etc.), (26) Presence of clinical signs of systemic infection (body temperature, heart rate, blood pressure, respiratory rate etc.), (27) Changes in 
biochemical parameters suggestive of organ (or tissue) infection, (28) Antibiotic regimen used, (29) Duration of the antibiotic therapy, (30) Outcomes of the antibiotic treatment (cure rate and mortality), (31) Adverse events rate and type, (32) Sensitivity to antibiotics, (33) Resistance to antibiotics.

Postoperative infection was considered to be any infection that occurred after a surgical intervention, with the development of symptoms during the postoperative recovery (while the patient was still hospitalized). Patients who developed symptoms after discharge from the hospital, after a certain period of time, were not considered as postoperative infections.

Infections on artificial materials (grafts) were considered if $C$. striatum was detected from the sample taken from the graft itself (central venous catheter, artificial valve, etc.) or from an adjacent tissue. The extraction of data was performed independently by each investigator.

For each study included (according to their design), all authors assessed four types of bias (i.e. selection, information, attrition and outcome reporting bias) separately and independently, and in case of disagreement, the final decision was made by the senior investigator (SJ). In addition, the uniformity of the tabular presentation of the included studies according to their distribution in different regions of the world in which they were conducted, was assessed.

The continuous variables (categorical) measured in this analysis were: total study duration, total number of patients, age of patients, maximal level of CRP, PCT and WBC during the infection and duration of antibiotic therapy, while the gender of patients, presence of hypertension, diabetes or malignancy, postoperative infection, infections on embedded artificial materials, site of $C$. striatum isolation, sampling method, method of $C$. striatum identification, morphological diagnoses that confirmed the invasive infections, presence of clinical signs of systemic infection, changes in laboratory parameters, antibiotic regimen used, outcomes of antibiotic treatment (cure rate and mortality), adverse events rate and type, sensitivity and resistance to antibiotics.

Each individual patient from the articles included in this review was considered as the unit of analysis.

The authors tried to reduce the percentage of missing data by searching them getting in contact with the researchers who conducted the original researches or by searching for data within the Results category in the ClinicalTrial.gov database. Within the Discussion section, the impact of missing data on the final results and possible conclusions was discussed. The heterogeneity evaluation could not be applied to this type of systematic review.

Basic methods of descriptive statistics (median and interquartile range for continuous variables and numbers and percentages for the categorical variables) were used to analyze and present the data.

\section{RESULTS}

\section{Characteristics of included studies and patients}

The literature search results are summarized in Figure 1. The final analysis covered a total of 42 studies (35 case reports ${ }^{1,2,12-44}$ and 7 case series ${ }^{5,9,45-49}$ ) reporting 85 individual cases that met the inclusion criteria and did not present exclusion criteria. The median age of the subject with the reported $C$. striatum invasive infection was 65 (IQR 51,72), with the oldest being 88 years old, while the youngest was 13 years old. About two/thirds of the cases were males $(57 / 67.1 \%), 27$ cases $(31.8 \%)$ were females, while in 1 case $(1.2 \%)$ the gender of the patient was not reported. The presence of hypertension was reported in 15 patients (17.6\%), 14 patients (16.5\%) had diabetes, while various types of cancers were reported in 13 cases (15.3\%). C. striatum caused postoperative infections (infections after invasive diagnostic and therapeutic procedures) in 24 patients $(28.2 \%)$. On the other hand, the causal role of C. striatum for infections of previously embedded artificial implants, such as orthopedic prostheses, artificial valves, etc., was reported in 19 patients $(22.3 \%)$. This systematic review article included patients with $C$. striatum invasive infection from European $(n=48)$, Asian $(n=17)$, North American $(n=16)$, South American $(n=3)$ and African continents $(n=1)$. Most of the cases were reported from Spain $(17 / 20 \%)$, followed by USA $(15 / 17.6 \%)$, India $(13 / 15.3 \%)$, France $(12 / 14.1 \%)$, Italy $(10 / 11.8 \%)$ and England (4/4.7\%). Korea, Japan and Brazil reported two patients each with invasive infections caused by $C$. striatum $(2 / 2.3 \%)$, while Morocco, Colombia, Greece, Northern Ireland, Canada, Turkey, Belgium and Netherland reported only one case each (1.2\%). The geographical distribution of included cases is presented in Figure 2. All patients involved were managed exclusively in hospitals. The longest followup last about 14 months $^{24}$. In five of six case series included, the attrition bias was evaluated as low, while in one study ${ }^{5}$, we estimated it to be medium, because the authors reported two lost-to-follow-up patients. Overview of the individual cases, as well as an assessment of the risk of bias in each study included are shown in Supplementary Table S1.

\section{Site and method of isolation of Corynebacterium striatum}

The presence of $C$. striatum was the most commonly confirmed in blood samples $(24 / 28.2 \%)$, followed by 


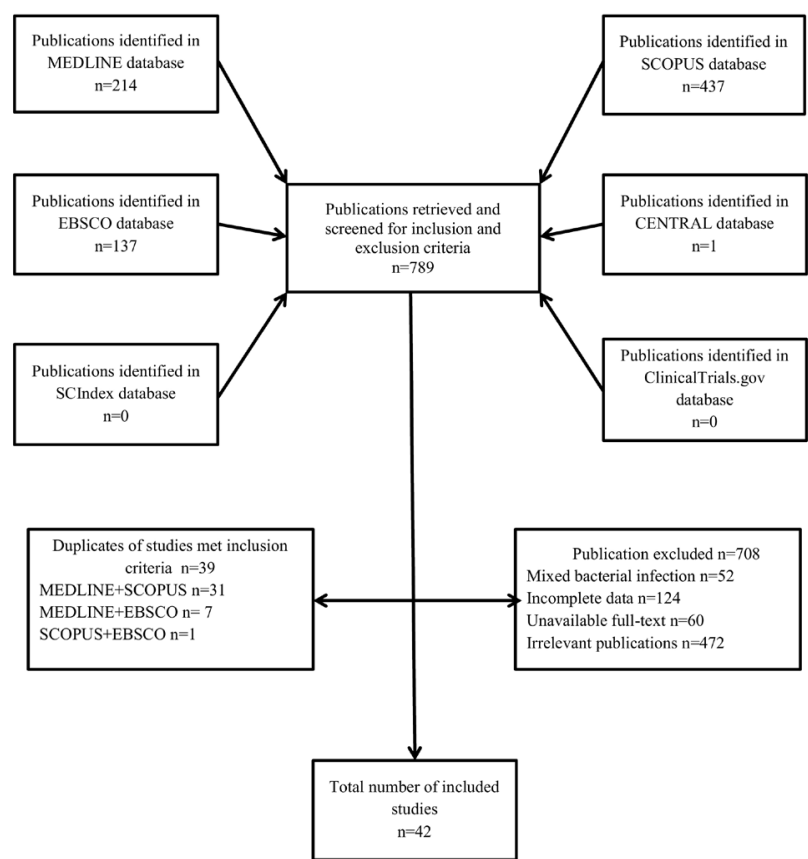

Figure 1 - The study selection flowchart.

intra- and postoperative samples from bone and joint infections $(13 / 15.3 \%)$, sputum $(12 / 14.1 \%)$, bronchial aspirate $(9 / 10.6 \%)$, ulcer aspirate $(5 / 5.9 \%)$, central venous catheter $(4 / 4.7 \%)$, wound aspirate $(4 / 4.7 \%)$, synovial fluid aspirate $(3 / 3.5 \%)$ nasal swab $(2 / 2.3 \%)$, tracheal aspirate $(2 / 2.3 \%)$, and broncho-alveolar lavage fluid $(2 / 2.3 \%)$. C. striatum was isolated in one patient each from pus, continuous ambulatory peritoneal dialysis fluid, pleural fluid, cerebrospinal fluid, wound swab, throat swab, urine, as well as from samples obtained from uterus, placenta,
IV catheter, lung biopsy, excised necrotic breast tissues, skin lesion biopsy, drainage of abscess and chest drainage.

C. striatum as a cause of infection was most often identified exclusively by biochemical methods (37/43.5\%), while additional confirmation using 16S rRNA gene sequencing was carried out in 7 (8.2\%) cases. On the other hand, mass spectrometry (in the vast majority of cases through the MALDI-TOF system) as a single method was used to identify C. striatum in 17 patients (20\%), while the causative agent was identified by a combination of biochemical methods and MALDI-TOF in 12 patients (14.1\%). 16S rRNA sequencing was the only method for identification of $C$. striatum in 9 patients $(10.6 \%)$, while in 2 cases $(2.3 \%)$ corynebacteria were identified by a combination of MALDI-TOF and 16S rRNA sequencing. Finally, in one patient (1.2\%) an invasive infection caused by $C$. striatum was confirmed by a combination of all three methods (biochemical+MALDI-TOF+16S rRNA sequencing).

\section{Susceptibility of $C$. striatum to antibiotics}

Antimicrobial susceptibility testing of $C$. striatum was performed in 64 patients $(75.3 \%)$, and the summarized susceptibility pattern of these isolates is depicted in Figure 3. When more than two isolates were reported to perform the susceptibility testing for specific antibiotics, all isolates were found to be susceptible to vancomycin (50/78.1\%), linezolid (30/46.9\%), teicoplanin (16/25\%), piperacillintazobactam $(8 / 12.5 \%)$, amoxicillin-clavulanate $(3 / 4.7 \%)$ and cefuroxime $(2 / 3.1 \%)$. On the other hand, all the isolates

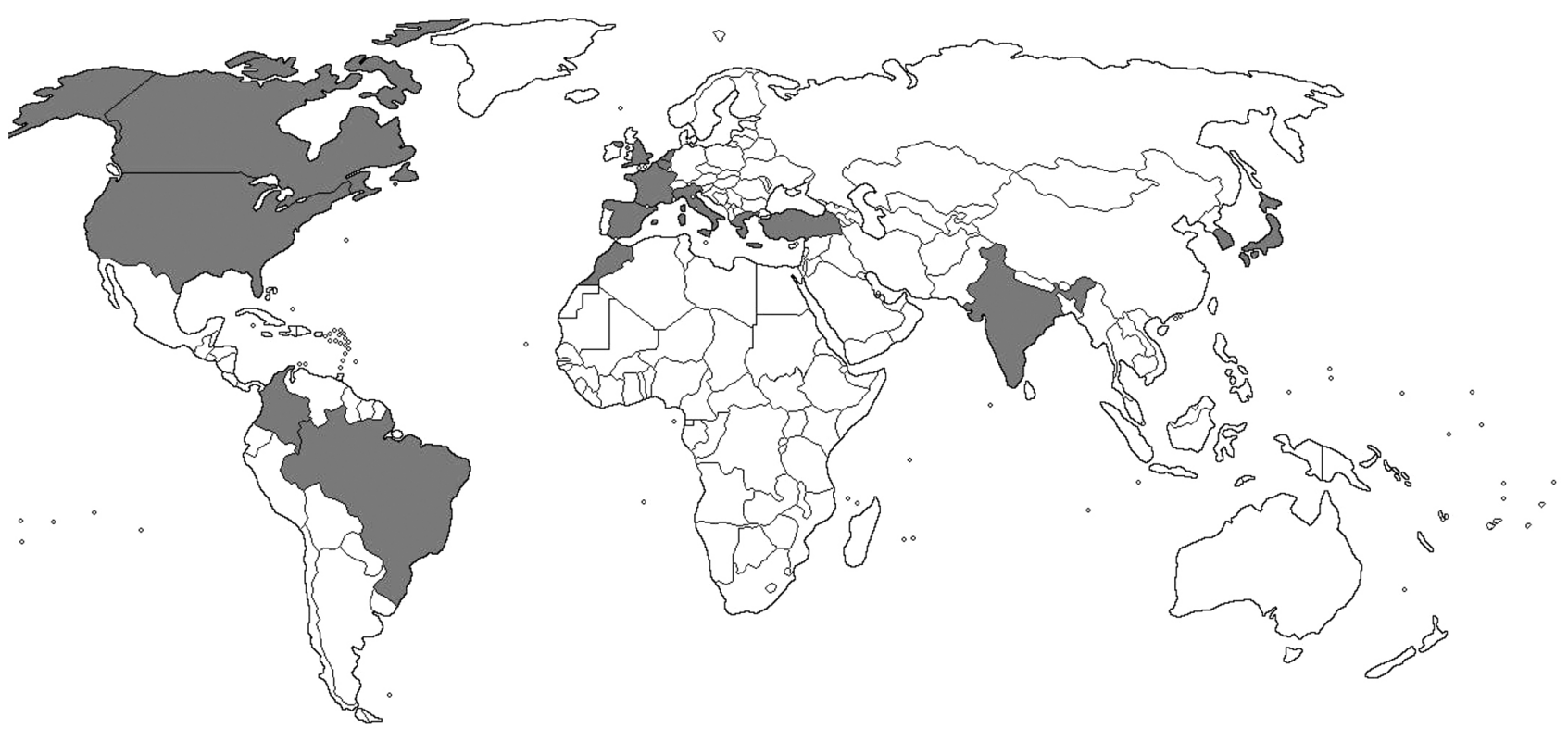

Figure 2 - Geographical distribution of Corynebacterium striatum studies. Countries in which C. striatum was isolated are shaded with grey color. 


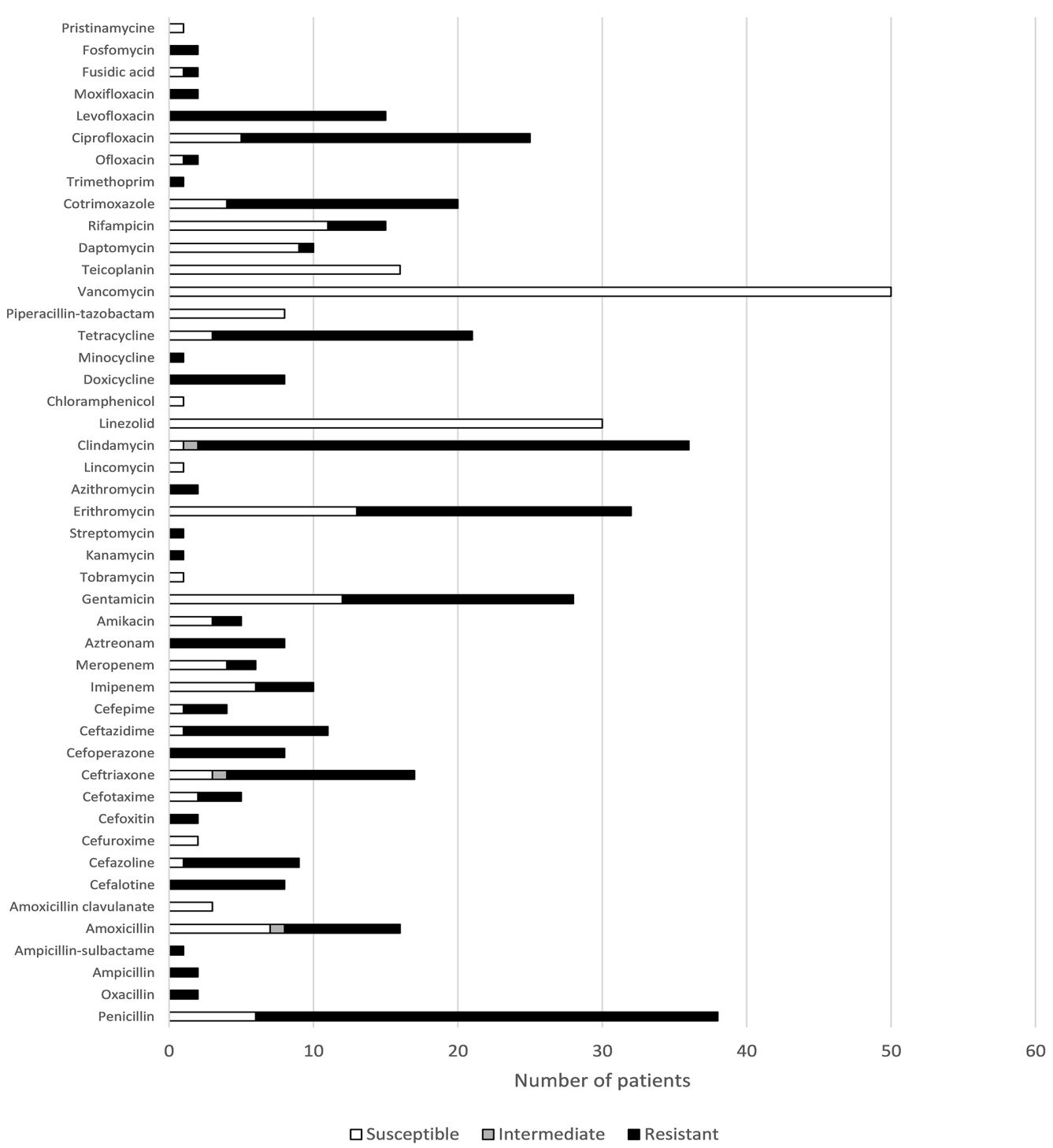

Figure 3 - Susceptibility of $C$. striatum to antibiotics.

tested to levofloxacin $(15 / 23.4 \%)$, cefalotin $(8 / 12.5 \%)$, cefoperazone $(8 / 12.5 \%)$, aztreonam $(8 / 12.5 \%)$, doxycycline (8/12.5\%), etC. were resistant. Isolates of $C$. striatum have also shown a high degree of resistance to penicillin (32 vs. 6 susC. isol.), cefazoline ( 8 vs. 1 susC. isol.), ceftriaxone (13 vs. 3 susC. isol), ceftazidime (10 vs. 1 susC. isolates), gentamycin (16 vs. 12 susC. isol.), erythromycin (19 vs. 13 susC. isol.), tetracycline (18 vs. 3 susC. isol., cotrimoxazole (16 vs. 4 susC. isol.), ciprofloxacin ( 20 vs. 5 susC. isol) and particularly to clindamycin (34 vs. 1 susC. isol).

\section{Antibiotic treatment of $C$. striatum infections}

Antibiotics used for treatment of invasive infections caused by $C$. striatum were reported in 83 patients $(97.6 \%)$. Treatment of $C$. striatum invasive infections in a slightly higher number of patients (44/53\%) was composed of a monotherapeutic antibiotic approach, while a combination of two or more antibiotics was used for the treatment of 39 patients (47\%). Vancomycin was the most commonly used antibiotic $(35 / 42.2 \%)$, followed by piperacillintazobactam $(20 / 24.1 \%)$, rifampicin $(11 / 13.2 \%)$ and amoxicillin (11/13.2\%) (Figure 4). Adverse effects of the applied antibiotics were reported only for patients with endocarditis, in whom a cutaneous rash occurred during the administration of ampicillin and gentamicin, leading to the replacement of this combination of antibiotics by vancomycin.

\section{Treatment outcomes}

More than half of the patients $(62.3 \%)$ experienced a complete recovery after the antibiotic treatment, while in 12 patients $(14.1 \%)$ a relevant clinical improvement was 


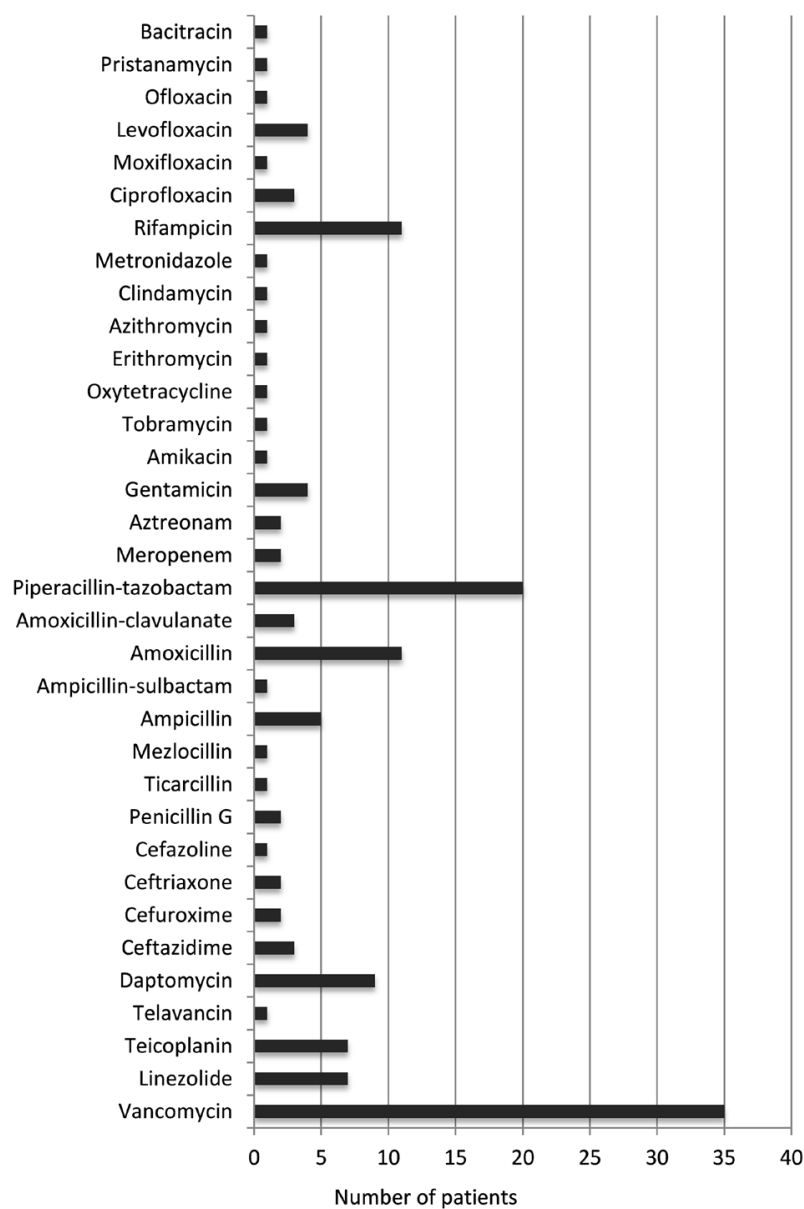

Figure 4 - Antibiotics used for the treatment of $C$. striatum infections.

reported after the administration of the antibiotic therapy. Death was reported in 15 patients (17.6\%), while failure of the antibiotic therapy resulted in recurrence, superinfection and limb amputation, reported in three patients (3.5\%) with orthopedic infections. Finally, the treatment outcome was not reported in two patients $(2.3 \%)$.

\section{DISCUSSION}

This systematic review confirmed the clinical relevance of $C$. striatum as a cause of various serious infections both in immunocompromised and immune competent inpatients. C. striatum was isolated from different types of biological and artificial samples and more frequently identified by specific biochemical tests in slightly more than $43 \%$ of the total number of patients. This bacterial species was also recovered from different infection sites, depending on the infected tissue. More than one isolate of C. striatum exhibited $100 \%$ susceptibility to vancomycin, linezolid, teicoplanin, piperacillin-tazobactam, amoxicillinclavulanate and cefuroxime, while strains of this bacterium showed a high degree of resistance to fluoroquinolones, the vast majority of $\beta$-lactams, aminoglycosides, macrolides, lincosamides and cotrimoxazole. Despite the use of antibiotics, fatal outcome was reported in almost $20 \%$ of the patients included in this study.

As a normal resident of the human skin and mucous membranes $C$. striatum is rarely a pathogenic microorganism. However, it seems that $C$. striatum has a particularly large pathogenic potential among non-diphtheritic bacteria from the genus Corynebacterium ${ }^{4}$. Microbiological in vitro studies have shown that the ability to form a biofilm on different abiotic surfaces stands out as the most significant virulence factor possessed by this bacterium ${ }^{50,51}$. C. striatum forms colonies equally efficiently on both, hydrophobic and hydrophilic surfaces, including polystyrene ureters. It could be expected that the $C$. striatum potential for biofilm formation is even higher in vivo due to the stimulatory effect of fibrinogen ${ }^{52}$. Biofilm makes bacteria more resistant to the action of immune system cells and antibiotics, which explains the growing number of bloodstream and catheter-related infections caused by $C$. striatum $^{50,51}$. Microbial biofilms play a key role in the pathogenesis of endocarditis ${ }^{53}$, which may explain the fact that this review includes as many as 10 reports of endocarditis caused by C. striatum $^{12,20,21,24-27,29,42,43}$. We also analyzed more than 10 cases with $C$. striatum prosthetitis, catheter-related infections and similar infections on embedded artificial devices and materials ${ }^{2,5,9,19,22,30}$, which are most likely a consequence of the aforementioned virulence factor available to this bacterium.

When it comes to the diagnostic method for identifying C. striatum, at least several examples of misidentification of this bacterium using biochemical methods have been reported in the literature. A particularly interesting case was reported in the intensive care unit of a clinic in Messina, Italy ${ }^{45}$. At the beginning of 2006, in eight patients with symptoms of severe infection, the causative agent was initially misidentified as Kocuria kristinae using the conventional Vitek 2 system (bioMérieux, Marcy L'Étoile, Lyon, France). Different types of biological samples ( 7 bronchial aspirates from 5 patients, 5 sets of blood cultures from two patients and one sample from a central venous catheter) were tested and the probability of identification was extremely high, of $99.9 \%$. However, $16 \mathrm{~S}$ rRNA sequencing was subsequently performed and all the 13 isolates were identified as $C$. striatum. The results of several studies ${ }^{54-56}$ indicate that gene sequencing methods are the most reliable for identifying C. striatum and should be the gold standard for the identification of this bacterium. MALDI-TOF is a slightly less accurate method than $16 \mathrm{~S}$ rRNA sequencing, but due to its cost-efficiency, simplicity and sufficient reliability, it can be used as an alternative 
method for the identification of C. striatum $^{54,55}$. Although cases of misdiagnosis of $C$. striatum using the VITEK 2 system (bioMerieux) can be found in clinical practice ${ }^{45}$, it could be considered that this method of identification of C. striatum is reliable after the inclusion of this pathogen in the database of the VITEK system (VITEK ${ }^{\otimes} 2$ ANC ID card $)^{57}$, especially in laboratories which do not dispose of equipments for the $16 \mathrm{~S}$ rRNA sequencing or the MALDI-TOF.

In recent years, $C$. striatum has been classified as multidrug-resistant, which is confirmed by the results of this systematic review article, due to a high degree of resistance showed to fluoroquinolones, the vast majority of $\beta$-lactams, aminoglycosides, macrolides, lincosamides and cotrimoxazole. Molecular mechanisms of resistance of $C$. striatum to these classes of antibiotics are already known. Resistance of C. striatum to fluoroquinolones is the most likely result of mutations at codons 87 and 91 of the QRDR gyrA gene ${ }^{58}$, while resistance of this bacterium to $\beta$-lactams is most likely a consequence of synthesis of $\beta$-lactamase ${ }^{3}$. The presence of multiresistance plasmid pTP10 explains the resistance that this bacterium shows to macrolides, tetracyclines and clindamycin ${ }^{59}$. However, despite the problem of resistance that $C$. striatum showed to a large number of antibiotics, our review confirmed a good in vitro susceptibility of this bacterium primarily to vancomycin, linezolid, teicoplanin and piperacillintazobactam, without any case of resistance. High resistance of $C$. striatum strains towards macrolides, lincosamides, fluoroquinolones, $\beta$-lactams and rifampicin on the one hand, respectively $100 \%$ susceptibility to vancomycin, daptomycin and linezolid, on the other hand, was also shown by Alibi et al. ${ }^{3}$.

This systematic review has several limitations. Firstly, a relatively small number of reported cases with invasive infections caused by $C$. striatum was analyzed. Secondly, 16S rRNA gene sequencing as the most accurate method for confirming C. striatum in different isolates was used in a minority of studies included in our review, implying a controversial reliability in terms of correct identification of this bacterium. Finally, in several analyzed reports, relevant information on the susceptibility of $C$. striatum to antibiotics, the applied antibiotic therapy, as well as on all the outcomes of treatment of the infections, were either missing or incompletely presented.

\section{CONCLUSION}

In conclusion, $C$. striatum could play a pathogenic role in causing serious invasive infections of various tissues accompanied by a significant mortality rate, predominantly in elderly patients and in those with significant level of immunosuppression, but also in immunocompetent hosts The gold standard for identifying $C$. striatum are the gene sequencing techniques, but the MALDI-TOF and Vitek 2 system can also be used with significant reliability, especially when there is a need for a rapid identification of the causative agent. Vancomycin, a broad-spectrum glycopeptide should be used as the antibiotic of choice, as monotherapy or in combination with piperacillin-tazobactam, depending on the type and severity of the infection. Alternatively, linezolid, teicoplanin or daptomycin may be used in severe infections, while amoxicillin-clavulanate may be used to treat only mild infections caused by $C$. striatum, if their potential use matches the results of the in vitro activity findings. Duration of antibiotic therapy should be adjusted according to the severity and anatomical site of the infection. During the administration of vancomycin, regular monitoring of renal function is necessary, especially in elderly patients. On the other hand, the use of linezolid for the treatment C. striatum infections requiring several months of treatment should be avoided, because the prolonged use of this antibiotic is accompanied by a higher risk of serious side effects.

\section{ACKNOWLEDGMENTS}

This study was partially financed by grant $\mathrm{N}^{\circ} 175007$, given by the Serbian Ministry of Education, Science and Technological Development.

\section{AUTHORS' CONTRIBUTIONS}

MM had idea for the article. MM, SS, AK, MM, JM and $\mathrm{MD}$, performed the literature search and data analysis. SJ critically revised the work.

\section{CONFLICT OF INTERESTS}

The authors declare no conflict of interests.

\section{REFERENCES}

1. Severo CB, Guazzelli LS, Barra MB, Hochhegger B, Severo LC. Multiple pulmonary nodules caused by Corynebacterium striatum in an immunocompetent patient. Rev Inst Med Trop Sao Paulo. 2014;56:89-91.

2. Yang HS, Kim YJ, Cho SY, Shin E, Lee HJ. Central venous catheter-related bloodstream infection by Corynebacterium striatum identified by $16 \mathrm{~S}$ rRNA and rpoB gene sequencing. Ann Lab Med. 2015;35:548-50.

3. Alibi S, Ferjani A, Boukadida J, Cano ME, Fernández-Martínez M, Martínez-Martínez L, et al. Occurrence of Corynebacterium 
striatum as an emerging antibiotic-resistant nosocomial pathogen in a Tunisian hospital. Sci Rep. 2017;7:9704.

4. Chen FL, Hsueh PR, Teng SO, Ou TY, Lee WS. Corynebacterium striatum bacteremia associated with central venous catheter infection. J Microbiol Immunol Infect. 2012;45:255-8.

5. Martínez-Martínez L, Suárez AI, Rodríguez-Baño J, Bernard K, Muniáin MA. Clinical significance of Corynebacterium striatum isolated from human samples. Clin Microbiol Infect. 1997;3:634-9.

6. Lee PP, Ferguson DA Jr, Sarubbi FA. Corynebacterium striatum: an underappreciated community and nosocomial pathogen. J Infect. 2005;50:338-43.

7. Leonard RB, Nowowiejski DJ, Warren JJ, Finn DJ, Coyle MB. Molecular evidence of person-to-person transmission of a pigmented strain of Corynebacterium striatum in intensive care units. J Clin Microbiol. 1994;32:164-9.

8. Brandenburg AH, van Belkum A, van Pelt C, Bruining HA, Mouton JW, Verbrugh HA. Patient-to-patient spread of a single strain of Corynebacterium striatum causing infections in a surgical intensive care unit. J Clin Microbiol. 1996;34:208994.

9. Noussair L, Salomon E, El Sayed F, Duran C, Bouchand F, Roux $\mathrm{AL}$, et al. Monomicrobial bone and joint infection due to Corynebacterium striatum: literature review and amoxicillinrifampin combination as treatment perspective. Eur J Clin Microbiol Infect Dis. 2019;38:1269-78.

10. McMullen AR, Anderson N, Wallace MA, Shupe A, Burnham CA. When good bugs go bad: epidemiology and antimicrobial resistance profiles of Corynebacterium striatum, an emerging multidrug-resistant, opportunistic pathogen. Antimicrob Agents Chemother. 2017;61:e01111-17.

11. Goldner NK, Bulow C, Cho K, Wallace M, Hsu FF, Patti GJ, et al. Mechanism of high-level daptomycin resistance in Corynebacterium striatum. mSphere. 2018;3:e00371-18.

12. Abi R, Ez-Zahraouii K, Ghazouani M, Zohoun A, Kheyi J, Chaib A, et al. Endocardite à Corynebacterium striatum chez un porteur de stimulateur cardiaque. Ann Biol Clin (Paris). 2012;70:329-31.

13. Beltrán-Arroyave C, Díaz-Díaz A, Loaiza-Díaz N. Osteomielitis crónica por Corynebacterium striatum en una adolescente. Rev Chilena Infectol. 2016;33:696-9.

14. Bhandari S, Meigh JA, Sellars L. CAPD peritonitis due to Corynebacterium striatum. Perit Dial Int. 1995;15:88-9.

15. Biswal I, Mohapatra S, Deb M, Dawar R, Gaind R. Corynebacterium striatum: an emerging nosocomial pathogen in a case of laryngeal carcinoma. Indian J Med Microbiol. 2014;32:323-4.

16. Chatzopoulou M, Koufakis T, Voulgaridi I, Gabranis I, Tsiakalou M. A case of fatal sepsis due to multidrug-resistant Corynebacterium striatum. Hippokratia. 2016;20:67-9.

17. Cone LA, Curry N, Wuestoff MA, O’Connell SJ, Feller JF. Septic synovitis and arthritis due to Corynebacterium striatum following an accidental scalpel injury. Clin Infect Dis. 1998;27:1532-3.

18. Cowling P, Hall L. Corynebacterium striatum: a clinically significant isolate from sputum in chronic obstructive airways disease. J Infect. 1993;26:335-6.

19. Daisuke U, Oishi T, Yamane K, Terada K. Corynebacterium striatum Bacteremia associated with a catheter-related blood stream infection. Case Rep Infect Dis. 2017;2017:2682149.

20. Elshibly S, Xu J, Millar BC, Armstrong C, Moore JE. Molecular diagnosis of native mitral valve endocarditis due to Corynebacterium striatum. Br J Biomed Sci. 2006;63:181-4.

21. Fernández Guerrero ML, Molins A, Rey M, Romero J, Gadea I. Multidrug-resistant Corynebacterium striatum endocarditis successfully treated with daptomycin. Int J Antimicrob Agents. 2012;40:373-4.

22. Fernández-Esgueva M, Pérez-Jimeno N, Roda-Rojo V, FerrerCerón I. Corynebacterium striatum prosthetic joint infection. Enferm Infecc Microbiol Clin. 2019;37:480-1.

23. Gehlhausen J, Strausburg MB, Gilbert KE, Alomari AK. Early botryomycosis-like plaques in the setting of Corynebacterium striatum septicemia. Int J Dermatol. 2019;58:969-71.

24. Houghton T, Kaye GC, Meigh RE. An unusual case of infective endocarditis. Postgrad Med J. 2002;78:290-1.

25. Jagadeeshan N, Jayaprakash S, Ramegowda RT, Manjunath CN, Lavanya V. An unusual case of Corynebacterium striatum endocarditis in a patient with congenital lymphedema and rheumatic heart disease. Indian Heart J. 2016;68 Suppl 2:S271-3.

26. Markowitz SM, Coudron PE. Native valve endocarditis caused by an organism resembling Corynebacterium striatum. J Clin Microbiol. 1990;28:8-10.

27. Marull J, Casares PA. Nosocomial valve endocarditis due to corynebacterium striatum: a case report. Cases J. 2008;1:388.

28. Roy M, Ahmad S. Rare case of Corynebacterium striatum septic arthritis. BMJ Case Rep. 2016;2016:bcr2016216914.

29. Rufael DW, Cohn SE. Native valve endocarditis due to Corynebacterium striatum: case report and review. Clin Infect Dis. 1994;19:1054-61.

30. Saito S, Kawamura I, Tsukahara M, Uemura K, Ohkusu K, Kurai H. Cellulitis and bacteremia due to Corynebacterium striatum identified by matrix-assisted laser desorption ionization-time of flight mass spectrometry. Intern Med. 2016;55:1203-5.

31. Savini V, Gherardi G, Favaro M, Fontana C, Marrollo R, Argentieri $\mathrm{AV}$, et al. About a bloodstream Corynebacterium striatum isolate. Folia Microbiol (Praha). 2013;58:451-3.

32. Scholle D. A spontaneous joint infection with Corynebacterium striatum. J Clin Microbiol. 2007;45:656-8.

33. Stone N, Gillett P, Burge S. Breast abscess due to Corynebacterium striatum. Br J Dermatol. 1997;137:623-5.

34. Superti SV, Martins DS, Caierão J, Soares F, Prochnow T, Cantarelli VV, et al. Corynebacterium striatum infecting a 
malignant cutaneous lesion: the emergence of an opportunistic pathogen. Rev Inst Med Trop Sao Paulo. 2009;51:115-6.

35. Tarr PE, Stock F, Cooke RH, Fedorko DP, Lucey DR. Multidrugresistant Corynebacterium striatum pneumonia in a heart transplant recipient. Transpl Infect Dis. 2003;5:53-8.

36. Tran TT, Jaijakul S, Lewis CT, Diaz L, Panesso D, Kaplan HB, et al. Native valve endocarditis caused by Corynebacterium striatum with heterogeneous high-level daptomycin resistance: collateral damage from daptomycin therapy?. Antimicrob Agents Chemother. 2012;56:3461-4.

37. Tumbarello M, Tacconelli E, Del Forno A, Caponera S, Cauda R. Corynebacterium striatum bacteremia in a patient with AIDS. Clin Infect Dis. 1994;18:1007-8.

38. Weiss K, Labbé AC, Laverdière M. Corynebacterium striatum meningitis: case report and review of an increasingly important Corynebacterium species. Clin Infect Dis. 1996;23:1246-8.

39. Westblade LF, Shams F, Duong S, Tariq O, Bulbin A, Klirsfeld $D$, et al. Septic arthritis of a native knee joint due to Corynebacterium striatum. J Clin Microbiol. 2014;52:1786-8.

40. Yoo G, Kim J, Uh Y, Lee HG, H GY, Yoon KJ. Multidrug-resistant Corynebacterium striatum bacteremia: first case in Korea. Ann Lab Med. 2015;35:472-3.

41. Selek MB, Atik TK, Bektöre B, Baylan O, Özyurt M. İmmün sistemi baskılanmış bir hastada Corynebacterium striatum'un etken olduğu pnömoni ve bakteriyemi olgusu. Gülhane Tip Derg. 2015;57:193-5.

42. Tibrewala AV, Woods CJ, Pyrgos VJ, Ruiz ME. Native valve endocarditis caused by Corynebacterium striatum. Scand J Infect Dis. 2006;38:805-7.

43. Keijman JM, Luirink MR, Ramsay G, Jacobs JA. Native valve endocarditis due to Corynebacterium striatum. Clin Microbiol Newsl. 2000;22:125-7.

44. Vandenbos F, Nicolaï C. Exacerbation aiguë des dilatations des bronches: imputabilité d'une infection à Corynebacterium striatum ? Rev Mal Respir. 2016;33:409-10

45. Iaria C, Stassi G, Costa GB, Biondo C, Gerace G, Noto A, et al. Outbreak of multi-resistant Corynebacterium striatum infection in an Italian general intensive care unit. J Hosp Infect. 2007;67:102-4.

46. Werth BJ, Hahn WO, Butler-Wu SM, Rakita RM. Emergence of high-level Daptomycin resistance in Corynebacterium striatum in two patients with left ventricular assist device infections. Microb Drug Resist. 2016;22:233-7.

47. Shariff M, Aditi A, Beri K. Corynebacterium striatum: an emerging respiratory pathogen. J Infect Dev Ctries. 2018;12:581-6.

48. Watkins DA, Chahine A, Creger RJ, Jacobs MR, Lazarus HM. Corynebacterium striatum: a diphtheroid with pathogenic potential. Clin Infect Dis. 1993;17:21-5.
49. Verroken A, Bauraing C, Deplano A, Bogaerts P, Huang D, Wauters G, et al. Epidemiological investigation of a nosocomial outbreak of multidrug-resistant Corynebacterium striatum at one Belgian university hospital. Clin Microbiol Infect. 2014;20:44-50.

50. Souza C, Simpson-Louredo L, Mota HF, Faria YV, Cabral FO, Colodette SD, et al. Virulence potential of Corynebacterium striatum towards Caenorhabditis elegans. Antonie Van Leeuwenhoek. 2019;112:1331-40.

51. Ramos JN, Souza C, Faria YV, Silva EC, Veras JF, Baio PV, et al. Bloodstream and catheter-related infections due to different clones of multidrug-resistant and biofilm producer Corynebacterium striatum. BMC Infect Dis. 2019;19:672.

52. Souza C, Faria YV, Sant'Anna LO, Viana VG, Seabra SH, Souza MC, et al. Biofilm production by multiresistant Corynebacterium striatum associated with nosocomial outbreak. Mem Inst Oswaldo Cruz. 2015;110:242-8.

53. Di Domenico EG, Rimoldi SG, Cavallo I, D’Agosto G, Trento E, Cagnoni $\mathrm{G}$, et al. Microbial biofilm correlates with an increased antibiotic tolerance and poor therapeutic outcome in infective endocarditis. BMC Microbiol. 2019;19:228.

54. Suh JW, Ju Y, Lee CK, Sohn JW, Kim MJ, Yoon YK. Molecular epidemiology and clinical significance of Corynebacterium striatum isolated from clinical specimens. Infect Drug Resist. 2019;12:161-71.

55. Gomila M, Renom F, Gallegos MC, Garau M, Guerrero D, Soriano JB, et al. Identification and diversity of multiresistant Corynebacterium striatum clinical isolates by MALDI-TOF mass spectrometry and by a multigene sequencing approach. BMC Microbiol. 2012;12:52.

56. Alibi S, Ferjani A, Gaillot O, Marzouk M, Courcol R, Boukadida J. Identification of clinically relevant Corynebacterium strains by Api Coryne, MALDI-TOF-mass spectrometry and molecular approaches. Pathol Biol (Paris). 2015;63:153-7.

57. Biomérieux. VITEK ${ }^{\circledR} 2$ ANC ID card: identification of anaerobic bacteria. [cited 2021 May 10]. Available from: https://www. biomerieux-diagnostics.com/vitek-2-anc-id-card

58. Sierra JM, Martinez-Martinez L, Vázquez F, Giralt E, Vila J. Relationship between mutations in the gyrA gene and quinolone resistance in clinical isolates of Corynebacterium striatum and Corynebacterium amycolatum. Antimicrob Agents Chemother. 2005;49:1714-9.

59. Tauch A, Krieft S, Kalinowski J, Pühler A. The 51,409-pb R-plasmid pTP10 from the multiresistant clinical isolate Corynebacterium striatum M82B is composed of DNA segments initially identified in soil bacteria and in plant, animal, and human pathogens. Mol Gen Genet. 2000;263:1-11. 